\title{
A SAÚDE DA PESSOA IDOSA NO CONTEXTO DA PANDEMIA PELO CORONAVÍRUS: CONSIDERAÇÕES PARA A ENFERMAGEM
}

\section{ELDERLY HEALTH IN THE CONTEXT OF THE CORONAVIRUS PANDEMIC: CONSIDERATIONS FOR NURSING}

\section{LA SALUD DE LOS ANCIANOS EN EL CONTEXTO DE LA PANDEMIA DEL CORONAVIRUS: CONSIDERACIONES PARA ENFERMERÍA}

Aline Miranda da Fonseca Marins ${ }^{1}$, Ana Maria Domingos ${ }^{2}$, Sabrina da Costa Machado Duarte ${ }^{3}$, Rafael Barroso Gaspar ${ }^{4}$, Simone Feliciano de Abreu $^{5}$, Lizandra Quintiliano de Carvalho ${ }^{6}$

\section{RESUMO}

Objetivo: Refletir sobre a saúde da pessoa idosa na pandemia pelo COVID - 19, para a elaboração de orientações de enfermagem, dirigidas a Atenção Primária à Saúde e à Assistência Hospitalar Especializada. Método: Análise e discussão à luz da produção científica sobre a COVID 19, pautando-se em indicadores epidemiológicos, clínicos, políticos, sociais e espirituais, considerados estruturantes do cuidado de enfermagem. Resultados: Identificou-se que a prática de enfermagem deve ser direcionada à pessoa idosa em três situações específicas e a seus desdobramentos: o idoso frágil e acamado na comunidade; o idoso autônomo e independente em isolamento social; o idoso em potencial risco de hospitalização. Conclusão: $O$ estudo identificou que idosos acamados, residentes em comunidades, compõem o grupo de alto risco dessa pandemia. Entende-se que os esforços para que a situação seja contida, requer empenho coletivo e conscientização da população e do cuidador do idoso. Sendo então, a articulação, os esforços e apelos realizados pelos profissionais de saúde, parte fundamental, para que ocorra a promoção da saúde e não só auxílio para resolutividade de comorbidades e recuperação dele. Ademais, também é dever do Estado acolher a população vulnerável, considerando os determinantes sociais implicados no processo de adoecimento a fim de minimizar os danos.

Descritores: Enfermagem Geriátrica; Idoso; Infecções por Coronavirus; Atenção Primária à Saúde; Hospitalização.

\section{ABSTRACT}

Objective: to reflect on elderly health during the COVID-19 pandemic and elaborate nursing guidelines focused on Primary Health Care and Specialized Hospital Care. Method: discussion and analysis of scientific production on COVID-19, based on epidemiological, clinical, political, social and spiritual indicators, which are considered to be the pillars of nursing care. Results: the nursing practice should be directed to the elderly in three specific situations and their consequences: the frail and bedridden elderly in the community; the autonomous and independent elderly in social isolation; the elderly at potential risk of hospitalization. Conclusion: the study shows that bedridden elderly people living in communities are the high-risk group of this pandemic. Efforts to contain the situation require collective commitment, awareness of the population, and attention of the elderly person's caregiver. Therefore, the articulation, efforts and appeals made by health professionals is fundamental for health promotion and not only assistance for the resolution of comorbidities and recovery. Moreover, the Government is responsible for embracing the vulnerable population, considering the social determinants involved in the process of illness in order to minimize the damage.

Descriptors: Geriatric Nursing; Aged; Coronavirus Infections; Primary Health Care; Hospitalization.

\section{RESUMEN}

Objetivo: reflexionar sobre la salud de los ancianos en la pandemia del COVID - 19, para la elaboración de pautas de enfermería dirigidas a la Atención Primaria de Salud y Atención Hospitalaria Especializada. Método: análisis y discusión a la luz de la producción científica sobre COVID 19, basado en indicadores epidemiológicos, clínicos, políticos, sociales y espirituales, considerados base de la estructura de la atención de enfermería. Resultados: se identificó que la práctica de enfermería debía dirigirse a los ancianos en tres situaciones específicas y sus consecuencias: los ancianos frágiles y acostados en la comunidad; los ancianos autónomos e independientes en aislamiento social; ancianos con riesgo potencial de hospitalización. Conclusión: el estudio identificó que los ancianos postrados en cama que viven en comunidades son el grupo de alto riesgo de esta pandemia. Se entiende que los esfuerzos para contener la situación requieren un compromiso y conciencia colectiva de la población y del cuidador de los ancianos. Así, la articulación, los esfuerzos y los llamamientos realizados por los profesionales de la salud, son una parte fundamental para la promoción de la salud y no sólo la asistencia para la resolución de las comorbilidades y la recuperación de las mismas. Además, también es deber del Estado acoger a la población vulnerable, teniendo en cuenta los determinantes sociales involucrados en el proceso de la enfermedad para minimizar el daño.

Descriptores: Enfermería Geriátrica; Anciano; Infecciones por Coronavirus; Atención Primaria de Salud; Hospitalización.

${ }^{1}$ Professor Adjunto do Departamento de Enfermagem Médico-Cirúrgica, Escola de Enfermagem Anna Nery, Universidade Federal do Rio de Janeiro (EEAN-UFRJ) - Brasil. ${ }^{2}$ Professora Associada do Departamento de Enfermagem em Saúde Pública da EEAN-UFRJ - Brasil. ${ }^{3}$ Professora Adjunta do Departamento de Metodologia da Enfermagem da EEAN-UFRJ - Brasil. ${ }^{4}$ Enfermeiro do Hospital Clementino Fraga Filho, da Universidade Federal do Rio de Janeiro. ${ }^{5}$ Enfermeira, coordenadora da Unidade da Terceira Idade no Programa de Assistência Integral à Pessoa Idosa no Instituto de Atenção Básica São Francisco de Assis UFRJ. ${ }^{6}$ Discente do Curso de Enfermagem da EEAN-UFRJ - Brasil. 


\section{INTRODUÇÃO}

Em dezembro de 2019, a cidade de Wuhan, na China, foi a primeira a relatar casos do SARSCoV-2 que faz parte da família Coronavírus, um vírus causador de infecções respiratórias. SARSCoV-2 é o agente causador da doença COVID-19, e em decorrência de sua alta taxa de transmissibilidade, a doença passou a ser enfrentada em vários países do mundo, tornandose, inicialmente, uma Emergência de Saúde Pública de Importância Internacional e, posteriormente, caracterizada como uma pandemia ${ }^{(1)}$.

A disseminação se dá por gotículas de saliva, espirro, tosse, catarro, contato pessoal como toque ou aperto de mão, contato com objetos ou superfícies contaminadas, acompanhado de contato com a boca, nariz ou olhos ${ }^{(1,2)}$. O período médio de incubação da infecção por Coronavírus é de 5.2 dias, mas pode chegar até 12.5 dias. A transmissibilidade dos pacientes infectados é em média de 7 dias após o início dos sintomas, no entanto, dados preliminares sugerem que a transmissão pode ocorrer mesmo com o indivíduo assintomático. Os sinais e sintomas comumente encontrados são: febre (temperatura axilar maior ou igual a $37,8 \circ \mathrm{C})$, tosse, dificuldade para respirar, dor muscular, fadiga, rinorréia, dor de garganta, dor de cabeça, dor no peito e confusão mental ${ }^{(3,4)}$.

Clinicamente, o início do quadro viral se caracteriza por uma síndrome gripal. Contudo, foi observado que casos iniciais leves e subfebris podiam evoluir para elevação progressiva da temperatura, ocasionando febre persistente ${ }^{(4)}$.

O risco de morte decorrente da COVID -19 aumenta com a idade do indivíduo e é mais frequente em pessoas com mais de 60 anos, especialmente aquelas com condições crônicas de saúde. ${ }^{(3,5)}$

Os idosos são o grupo mais vulnerável a todas as doenças infecciosas e essa realidade os deixa na faixa dos que mais têm complicações entre os infectados pelo novo coronavírus. Algumas doenças agravam ainda mais a chance de a população idosa a desenvolver estados avançados do coronavírus, como problemas no pulmão, obesidade e diabetes mellitus ${ }^{(5)}$. A letalidade é, significativamente, maior em idosos e em indivíduos com comorbidades (câncer 5,6\%, hipertensão arterial $6 \%$, doença respiratória crônica $6,3 \%$, diabetes mellitus $7,3 \%$ e doença cardiovascular $10,5 \%)^{(6)}$.

Idosos, especialmente portadores de comorbidades como diabetes, hipertensão arterial, doenças do coração, pulmão e rins, doenças neurológicas, em tratamento para câncer, portadores de imunossupressão entre outras, e aqueles com mais de 80 anos e portadores de síndrome de fragilidade, necessitam adotar medidas de restrição de contato social. O atendimento às pessoas idosas deve ser, preferencialmente, em domicílio, evitando-se a exposição coletiva em serviços de saúde ${ }^{(7)}$.

Nesse contexto, a APS/ESF é a porta de entrada do Sistema Único de Saúde. Durante surtos e epidemias, a APS/ ESF tem papel fundamental na resposta global à doença em questão, com grande potencial de identificação precoce de casos graves que devem ser manejados em serviços especializados $^{(13)}$.

Acerca disso, há implicações importantes para a organização dos serviços de saúde e das ações para o controle da doença, incluindo prevenção, diagnóstico e tratamento ${ }^{(3)}$. Em razão da letalidade muito mais elevada da COVID-19 entre os idosos (pessoas com 60 anos ou mais), deve-se priorizá-los para atendimento ${ }^{(13)}$.

No que concerne à assistência especializada, com a evolução da doença, as pessoas infectadas apresentam progressiva dificuldade respiratória, síndrome do desconforto respiratório agudo ou complicações com risco de vida, levando a uma necessidade de cuidados intensivos. Como em muitos casos, os idosos já possuem doenças subjacentes e, portanto, costumam apresentar imunossupressão, os sintomas podem aparecer de forma atípica, com risco comum para o mau prognóstico para a Covid-19 $9^{(8)}$.

Ressalta-se assim, o imperioso papel que as equipes de saúde possuem no enfrentamento dessa pandemia (COVID-19), especialmente, os profissionais de enfermagem, na prestação de cuidados à saúde da população em geral e, especialmente, a saúde da população idosa.

Os cuidados de enfermagem iniciam-se, desde a porta de entrada das Unidades Básicas de Saúde, na realização de triagem e acolhimento a usuários e casos suspeitos de infecção pelo Covid19 , na consulta de enfermagem, propriamente dita, nas orientações e condutas para o isolamento domiciliar, nos encaminhamentos, na sala de vacinação, na atenção especializada à saúde dos idosos, no cuidado à demanda programada, na visita domiciliar, na vigilância dos casos suspeitos, nos protocolos para a adequada higienização das mãos e uso de equipamentos de proteção individual (EPIs) e procedimentos de limpeza da unidade de saúde ${ }^{(9)}$ até a assistência hospitalizada especializada ${ }^{(8)}$.

Pelo exposto, foi delineado o seguinte objetivo de estudo: refletir sobre a saúde da pessoa idosa na pandemia pelo COVID-19, para a elaboração de orientações de enfermagem dirigidas a Atenção Primária à Saúde e à Assistência Hospitalar Especializada.

\section{MÉTODO}

Trata-se de um estudo de reflexão oriundo da análise e discussão dos autores à luz da produção científica atual sobre a COVID-19, disponível, especialmente, em meio eletrônico, considerando-se as especificidades da pessoa idosa, com vistas à formulação de conteúdos para infográficos e outros materiais informativos, a partir da necessidade apresentada pela própria população idosa e o compromisso com a extensão universitária, reforçada nas ações de extensão direcionadas à área especializada / hospitalar e atenção primária à saúde, especificamente, no Projeto de Extensão Cuidando do Idoso e de seu Cuidador (PROECIC - EEAN - UFRJ) e no Programa 
de Assistência Integral à Pessoa Idosa (PAIPI HESFA - UFRJ). Cabe reforçar que esse estudo de reflexão é aqui acentuado como estruturante do processo de construção das orientações de enfermagem à pessoa idosa/família, em ambos os contextos, atenção hospitalar especializada e atenção primária à saúde, tendo em vista os desafios e tendências suscitados pela pandemia. Atentou-se, ainda, para os indicadores: epidemiológicos, clínicos, políticos, sociais e espirituais, que permeiam as orientações de enfermagem.

\section{RESULTADOS E DISCUSSÃO}

\section{Orientações de enfermagem à pessoa idosa frágil e acamada na comunidade}

No caso da infecção por COVID-19, o desafio é a detecção precoce de sintomas para se antecipar ao agravamento rápido do quadro. Nesse aspecto, a pessoa idosa acamada é ainda mais vulnerável, pela alta transmissibilidade do vírus, o que acresce as demandas do idoso e/ou agravamento do seu padrão de saúde.

A COVID-19 é semelhante a uma gripe comum: primeira fase epidemiológica "casos importados", pessoas regressando de países com epidemia; segunda fase transmissão local e identificação da pessoa que transmitiu; terceira fase transmissão comunitária e proliferação dos casos e não identificação da fonte ${ }^{(7)}$. Nesse sentido, vale o seguinte questionamento: por que o idoso se torna população de risco, no contexto da infecção pelo SARS-CoV-2 ?

Uma das causas é a fragilidade, uma síndrome clínica caracterizada pela diminuição da força, resistência e função fisiológica, aumentando a vulnerabilidade de um indivíduo ao desenvolvimento de maior dependência e/ou morte ${ }^{(10)}$.

De acordo com a Política Nacional de Saúde da Pessoa Idosa ${ }^{(11)}$, Idoso frágil ou em situação de fragilidade é aquele que vive em Instituição de Longa Permanência para Idosos (ILPI); encontra-se acamado; esteve hospitalizado recentemente por qualquer razão; apresenta doenças sabidamente causadoras de incapacidade funcional (acidente vascular encefálico, síndromes demenciais, outras doenças neurodegenerativas, etilismo, neoplasia terminal, amputações de membros); encontra-se com, pelo menos, uma incapacidade funcional básica ou viva situações de violência doméstica.

Dessa forma, é mister apontar a problemática do idoso acamado, residindo em comunidade, pois a demanda de atenção à saúde é motivo de preocupação para os que envelhecem, para as famílias e para as equipes de saúde na cobertura assistencial contra a COVID 19.

Como exemplo de necessidades específicas, pode-se citar que um idoso acamado em domicílio vivencia a privação da sociabilidade. É sabido que as relações sociais são benéficas, porém na COVID19 , os cuidados adicionais são vitais e privam o idoso de contatos sociais presenciais. Por isso, é importante enfatizar que o ambiente físico do idoso deve ser seguro, para além de ser estimulante, de estar entre amigos e com a família ${ }^{(12)}$.

A Política Nacional de Saúde da Pessoa Idosa $^{(11)}$ trata da saúde adequada e digna aos idosos. Nesse sentido, a Atenção Primária/Estratégias de Saúde da Família é responsável por atender, em domicílio, a população, sendo a porta de entrada do Sistema Único de Saúde (SUS), na coordenação do cuidado nos diferentes níveis de atenção à saúde. Destacase, desse modo, a importância das equipes de saúde da família, no manejo de medidas de prevenção comunitária voltadas à população idosa ${ }^{(13)}$.

A equipe de enfermagem deve acompanhar os idosos frágeis e suas famílias cuidadoras orientando-os a realizar a educação comportamental, apresentar a etiqueta respiratória e orientar a manutenção do isolamento domiciliar. Na presença de suspeita da síndrome gripal, devese evitar encaminhar o idoso frágil para atendimento de urgência (pronto socorro, UPA, etc), onde o risco de contaminação é elevado ${ }^{(9)}$.

As visitas domiciliares são uma excelente estratégia para orientar os usuários, especialmente, orientações relacionadas à Covid19. Antes de ir ao domicílio, a equipe de saúde deve tentar se comunicar com a família para conhecer a possibilidade de casos com síndrome gripal, realizar mapeamento das visitas e, em casos de realização de procedimentos no domicilio, o profissional precisar levar os EPIs e colocar antes de entrar na residência e ao sair retirá-los com técnica asséptica e colocá-los em uma sacola plástica ${ }^{(9)}$.

\section{Orientações de enfermagem à pessoa idosa autônoma e independente em isolamento social}

Especificamente, nessa abordagem, considera-se o idoso autônomo e independente em condição de isolamento social, cujo manejo das respostas tem duplo desafio: primeiro auxiliá-lo a entender seu protagonismo no gerenciamento do seu autocuidado frente à disseminação da COVID19 e o segundo é atentar para as manifestações iniciais da doença.

Vale destacar, o manejo das orientações para a pessoa idosa em isolamento social, sendo fundamental o entendimento de que as especificidades do envelhecimento devem ser consideradas no planejamento das ações/intervenções pelas equipes de saúde da família.

Nesse sentido, o profissional de enfermagem pode traçar as seguintes estratégias e orientações junto aos idosos e sua rede de suporte social: Estimular a manutenção das Atividades da Vida Diária (AVDs); Incentivar a participação da rede social de apoio que visa, prioritariamente, à redução das chances de contaminação da COVID19; Enfatizar a necessidade de restrição de atividades que exponham os idosos a risco de contaminação (nesse caso, evitar sair de casa é a primeira regra); Explicar a importância da higienização das mãos com uso de água e sabão ou o álcool em gel. No tocante ao ambiente domiciliar, 
se possível, manter uma distância mínima de 1 metro ( 3 pés) entre o idoso e qualquer pessoa que esteja tossindo ou espirrando; Explicar a etiqueta respiratória que se compõe de medidas como cobrir a boca e o nariz com o cotovelo dobrado ou com um lenço de papel ao tossir ou espirrar e que deve ser descartado, imediatamente. Manter o ambiente domiciliar bem ventilado; limpar e desinfetar, com álcool 70\%, objetos e superfícies tocadas com frequência (cabeceira da cama, controle remoto, maçanetas, celulares, bengalas etc.); Manter alimentação saudável e boa ingestão de líquidos ${ }^{(7)}$.

Cabe salientar que o isolamento social pode provocar impacto nos níveis de humor, principalmente em idosos que moram só, em decorrência da mudanças nos hábitos de vida e nas relações sociais, pelo distanciamento de familiares e amigos. Sendo assim, é importante que o enfermeiro possa propor ao idoso e sua família cuidadora: estímulo na realização de atividades lúdicas, recreativas e físicas de baixo impacto, que na situação de isolamento se constituem em uma intervenção capaz de promover o conforto e bemestar. São também opções de atividades a serem estimuladas: Televisão, Rádio, computador, Jogo de Baralho, Jogo de Dominó, Jogo de Dama, Dança; Crochê, Bordado, Pintura, Tricô; Leitura de jornais, revistas, livros, palavras cruzadas ${ }^{(14)}$.

Atividades que estimulam o lazer, criatividade e novas experiências ganham destaque como, por exemplo: cuidar das plantas, organizar algum cômodo da casa, compartilhar vídeo ou fotos do seu dia a dia com aqueles que estão distantes, fazer uma chamada de vídeo com seus familiares, combinar com amigos para assistirem a um mesmo filme e depois discutirem sobre ele, montar um quebra-cabeças, fazer uma lista de lugares para visitar após o isolamento social, assistir um concerto musical on-line e visitar um museu virtual ${ }^{(15)}$.

É de suma importância e, também um cuidado ao idoso, o gerenciamento de pensamentos catastróficos, especialmente, numa pandemia, na qual a sociedade vivencia o isolamento social / distanciamento social. Esses tipos de pensamentos referem-se às tendências em interpretar a realidade a partir da imaginação dos piores desfechos. Uma forma de gerenciar esses pensamentos é o gerenciamento emocional: identificando os estados emocionais, identificando os pensamentos que antecedem os estados emocionais, rastrear possível utilização de lente catastrófica, questionar pensamentos catastróficos e reformular os pensamentos de forma mais equilibrada ${ }^{(15)}$.

Além das medidas com a higiene pessoal, com os objetos e afins, o exercício da espiritualidade / religiosidade é um cuidado essencial e que pode ser estimulado no enfrentamento da pandemia pelo COVID-19. A experiência religiosa pode ser especialmente útil para lidar com crises e traumas quando os indivíduos não dispõem de outros recursos que possam contribuir para esse suporte ${ }^{(16)}$.
Dessa forma, assistir virtualmente / on line, a missas, pregações, cultos, orações etc., respeitando as crenças pessoais de cada idoso é um cuidado a ser incentivado e orientado pelo enfermeiro. Há necessidade de ampliar o desenvolvimento de habilidades de pessoas mais velhas para uso de tecnologias, visando à informação, à comunicação e à participação social $^{(17)}$.

No contexto da utilização da tecnologia para a comunicação, no atual momento de enfrentamento da infecção pelo COVID-19, onde os contatos sociais, físicos e presenciais devem ser evitados, muitos pedidos e compras são realizados via online e entregues nas moradias. Dessa forma, como medida de prevenção, deve ser recomendado que, em casos de serviços "delivery", todas as embalagens sejam higienizadas. Nos casos excepcionais, como, por exemplo, idas ao mercado, recomenda-se priorizar os horários menos explorados $^{(18)}$.

Outro manejo de cuidado de enfermagem refere-se a idosos que convivem com familiares. Caso esses últimos apresentarem sintomas de gripe, devem evitar, imediatamente, o contato com o idoso. Reforça-se a necessidade de vacinação do idoso e seus contactantes. A vacinação é a forma mais eficaz de prevenção contra a gripe e suas complicações. A vacina é segura e é considerada uma das medidas mais eficazes para evitar casos graves e óbitos por gripe. A constante mudança dos vírus influenza requer um monitoramento global e frequente reformulação da vacina contra a gripe. Em razão dessa mudança dos vírus, é necessária a vacinação anual contra a gripe. A vacina não tem eficácia contra o coronavírus, mas protege contra outros tipos de vírus da gripe ${ }^{(19)}$.

Além das medidas já mencionadas, como medida de prevenção, o enfermeiro pode recomendar ao idoso e familiares: individualizar toalhas, sabonete, talheres, copos e pratos. Suspender visitas, optar por vídeochamadas, chats e telefonemas. Manter pelo menos um metro de distância da pessoa que esteja com os sintomas. Não compartilhar camas. Se possível, individualizar os quartos. Higienizar banheiros com água sanitária ${ }^{(13)}$.

A equipe de enfermagem e o enfermeiro devem atentar-se para a vigilância nos casos suspeitos de Covid-19. Caso o idoso venha a apresentar febre e tosse, o manejo clínico prevê o seu acompanhamento a cada 48 horas, mediante visita domiciliar com medidas de precaução de contato ou por contato telefônico. No caso de o quadro apresentar algum novo sintoma, a orientação é manter o idoso em quarto isolado e bem ventilado até o fim do período sintomático, manutenção da higiene respiratória e de hábitos saudáveis referentes à alimentação e hidratação ${ }^{(13)}$. No agravamento das condições clínicas a família entrará em contato imediato com a equipe de saúde que fará os encaminhamentos pertinentes ${ }^{(7)}$. Os familiares, colaboradores e cuidadores de idosos devem redobrar os cuidados com a higiene pessoal e limpeza da moradia ${ }^{(18) .}$ 
Na atenção primária a saúde, recomenda-se manter um mapa de atualização dos casos leves de Covid-19 para a vigilância ativa que é a principal ferramenta de manejo dos casos de prevenção de transmissão comunitária ${ }^{(9)}$.

Os cuidadores são elementos chave no cuidado ao idoso em domicílio, seja ele cuidador familiar e/ou não familiar (cuidador formal). Quadros demenciais são comuns na população idosa, sendo as doenças neurodegenerativas causadoras de incapacidades funcionais que, por sua vez, geram dependência, sendo o cuidador a pessoa mais importante na vida de um portador de demência. No momento pandêmico, o cuidador, deve ser orientado quanto às medidas preventivas de contaminação, tanto ao paciente quanto a si próprio $^{(20)}$

Diante dos diversos desafios postos pela pandemia da COVID-19, é necessário vislumbrar estratégias de enfrentamento e possibilidades para ações junto aos idosos saudáveis e familiares. No âmbito profissional, salientam-se as plataformas de educação a distância, com disponibilidade de diversos cursos online gratuitos sobre a temática COVID-19, propostos por Instituições de Ensino Públicas e privadas, assim como Governo Federal. Além disso, diversos periódicos científicos abriram chamadas específicas para a temática e publicaram artigos com diversas vertentes que envolvem o período pandêmico ${ }^{8)}$.

\section{Orientações de enfermagem a pessoa idosa em potencial risco de hospitalização}

O risco de desenvolver incapacidade se acentua, em momentos de crise, logo, a presença de processos agudos que requerem a hospitalização, é um momento delicado para muitos idosos $^{(21)}$.

No que tange à assistência hospitalar especializada, é importante considerar que os efeitos do envelhecimento ocasionam condições anatômicas e fisiológicas que potencializam o surgimento e desenvolvimento de problemas respiratórios. Nota-se que os tecidos conectivos responsáveis pela respiração estão mais fracos, a retração elástica dos pulmões, durante a expiração está diminuída (diminuição elástica do colágeno e elastina), o que requer o uso ativo da musculatura acessória. Além disso, os alvéolos estão com a elastina diminuída, desenvolvem tecido fibroso e contêm poucos capilares funcionantes. Reduz-se a capacidade vital e aumenta o volume residual. Como efeito final há diminuição da troca de ar e acúmulo de secreções nos pulmões ${ }^{(22)}$.

Ainda sobre os efeitos do envelhecimento, os diferentes padrões de temperatura corpórea podem encobrir as febres que, muitas vezes, aparecem em níveis atípicos, o que pode gerar confusão quanto à evolução de uma infecção respiratória. Em geral, os idosos têm uma margem de reserva fisiológica menor e são menos capazes de compensar e se adaptar a mudanças fisiológicas, por isso, a infecção é uma situação problemática nesses indivíduos. Os sinais e sintomas de pneumonia podem ser alterados e uma pneumonia severa, possivelmente, se faz presente sem a evidência de sintomas ${ }^{(22)}$. Em pessoas infectadas pelo COVID-19, o quadro de pneumonia bilateral foi comumente observado em exames de imagem. Somado a isso, outras complicações observadas foram a Síndrome Respiratória Aguda Grave (SRAG), seguida da lesão cardíaca aguda e infecções secundárias ${ }^{(2)}$.

Nesse sentido, é primordial ressaltar a atenção da equipe de enfermagem, na assistência ao idoso com COVID-19: a) Suspeitar de infecção, quando qualquer modificação abrupta, inexplicada, for detectada na função física e mental do idoso, b) Observar atentamente e registrar a temperatura corporal, pois as alterações no padrão dessa temperatura, em idosos, pode ocasionar pouca ou nenhuma febre, mascarando, por exemplo, um quadro de pneumonia, c) Observar e registrar confusão mental, agitação e mudanças comportamentais.

De acordo com a Portaria no 758, de 9 de abril de $2020^{(23)}$, ficam definidos os procedimentos para o registro obrigatório de internações hospitalares dos casos suspeitos e confirmados de COVID-19, nos estabelecimentos de saúde públicos e privados que prestam serviços no SUS. Ainda de acordo com essa portaria, o registro obrigatório contará, no mínimo, com as seguintes informações: I. O número de internações de pacientes em leitos clínicos/enfermaria e/ou leitos intensivos (UTI) com suspeita ou confirmação de COVID-19; II. O número de altas hospitalares (saídas) de pacientes suspeitos e confirmados para COVID-19; e III. Quantidade de leitos clínicos/enfermaria e/ou leitos intensivos (UTI) existentes no estabelecimento de saúde disponíveis para COVID19.

Considerando a organização emergencial da rede de atenção à saúde, pesquisadores da UFRJ, UERJ e FIOCRUZ, recomendaram, conjuntamente em Nota Técnica ${ }^{(24)}$, a adoção estratégica de três medidas que buscam aumentar a velocidade de respostas e a tomada de decisão apoiada em evidências e, consequentemente, garantir a assistência à saúde com maior segurança e resolutividade, direcionadas ao Estado do Rio de Janeiro. Essas três medidas seriam: Criação de uma sala de situação: para o monitoramento, coordenação da comunicação/ informação e gerenciamento das demandas do setor de saúde, do Estado do Rio de Janeiro); Articulação e interação de atores e setores: por meio da estruturação da rede de atenção à saúde pública e privada vinculada ao SUS, a fim de organizar a oferta de serviços à população, com suporte de setores estratégicos e entidades de classe, em ações de curto, médio e longo prazo; Plano Estratégico Emergencial: para Assistência à Saúde na pandemia pelo novo coronavírus / COVID-19. Essencialmente, o plano deverá elencar ações que respondam às seguintes perguntas: o quê, onde, quem e como, incluindo recursos financeiros.

Além das medidas mencionadas, cabe ressaltar a necessidade de instrumentalização, capacitação e especialização dos profissionais de 
saúde para atender a clientela idosa, visto ser um grupo populacional que requer cuidados direcionados, dada a sua especificidade e complexidade de atendimento. "Os profissionais enfermeiros estarão mais bem preparados quanto mais notáveis forem os conhecimentos relativos ao processo de envelhecimento, bem como o olhar atento às situações e condições valorizadas pelos próprios idosos"(25).

\section{CONSIDERAÇÕES FINAIS}

Esse estudo possibilitou refletir que é notório que idosos acamados residentes em comunidades são o grupo de alto risco dessa pandemia. Dessa forma, entende-se, que os esforços para que a situação seja contida requer empenho coletivo e conscientização da população e do cuidador do idoso. $O$ ato de ficar em casa não é só para própria segurança, mas também para a proteção daqueles que são mais vulneráveis. Sendo então, a articulação, os esforços e apelos realizados pelos profissionais de saúde, são fundamentais para que, em sua maioria, ocorra a promoção da saúde e não só o auxílio para resolutividade de comorbidades e recuperação dele.

Nesse momento, também é dever do Estado acolher a população, considerando os determinantes sociais implicados no processo de adoecimento, principalmente, os que se encontram em situação vulnerabilidade, a fim de minimizar os danos. Sobre as populações mais vulneráveis, existem possibilidades reais da disseminação desse vírus, pois vivem em situações de aglomerações, sem saneamento básico, entre outros problemas. Por isso, deve-se exigir das autoridades públicas formulação de estratégias de proteção aos grupos mais vulneráveis.

Faz-se mister ressaltar o primoroso papel que a Atenção Primária à Saúde possui na organização dos serviços de saúde, com destaque na sua atuação e delineamento de estratégias para o enfrentamento na Covid-19.

Por fim, no contexto da pandemia pelo COVID-19, embora haja evidências de que a população idosa, especialmente os idosos frágeis, estejam entre o grupo de maior risco, reforça-se que há necessidade de estudos aprofundados abordando, especificamente, as implicações dessa pandemia no contexto da saúde dessa população, assim como, a capacitação de profissionais para atuarem junto a essa clientela.

\section{REFERÊNCIAS}

1- World Health Orgazization (WHO). Coronavirus disease (COVID-19) pandemick. 2020 [citado em 22 mar 2020]. Available in: https://www.who.int/emergencies/diseases/novelcoronavirus-2019

2- Brasil, Ministério da Saúde. O que é coronavírus? 2020a [citado em 22 mar 2020]. Available in: https://coronavirus.saude.gov.br/

3- Lloyd-Sherlock P, Ebrahim S, Geffen L, McKee M. Bearing the brunt of covid-19: Older people in low and middle income countries. BMJ 2020;368:m1052. DOI: 10.1136/bmj.m1052
4- Brasil, Ministério da Saúde. Protocolo de manejo clínico para o novo coronavírus (2019nCoV). Brasília, DF: Ministério da Saúde; 2020b.

5- Brasil, Sociedade Brasileira de Geriatria e Gerontologia (SBGG). Sinais de alerta nos idosos sobre a COVID-19. 2020c [citado em 22 mar 2020]. Available in: https://sbgg.org.br/sinais-de-alertanos-idosos-sobre-a-covid-19/

6- Universidade Federal de Minas Gerais, Núcleo de Geriatria e Gerontologia da UFMG. A pandemia por coronavírus e o idoso. Belo Horizonte: UFMG; 2020.

7- Brasil, Sociedade Brasileira de Geriatria e Gerontologia (SBGG). Posicionamento sobre COVID-19. 2020d [citado em 22 mar 2020]. Available in: https://sbgg.org.br/posicionamentosobre-covid-19-sociedade-brasileira-de-geriatria-egerontologia-sbgg-atualizacao-15-03-2020

8- Associação Brasileira de Enfermagem (ABEn). Enfermagem gerontológica no cuidado do idoso em tempo da COVID 19. Brasília, DF: ABen; 2020. Ebook

9- Comitê Interno de Acompanhamento das Ações de Prevenção e Enfrentamento do Novo Coronavírus. (Comitê Covid-19). Orientações para organização e fluxos nas unidades básicas de saúde em tempo de coronavírus. Belo Horizonte: Comitê Covid-19; 2020.

10- Morley JE, Vellas B, Kan GA, Anker SD, Bauer $J M$, Bernabei $R$, et al. Frailty consensus: $A$ call to action. J Am Med Dir Assoc. 2013;14(6):392-7. DOI: 10.1016/j.jamda.2013.03.022

11- Brasil, Ministério da Saúde. Portaria $n^{\circ} 2.528$ de 19 de outubro de 2006. Aprova a Política Nacional de Saúde da Pessoa Idosa. Diário Oficial da União 2006.

12- NERI AA. O envelhecimento no universo do trabalho: Desafios e oportunidades depois dos 50 anos. In: Barros JC (Org). Empreendedorismo, trabalho e qualidade de vida na terceira idade. São Paulo: Edicon; 2009.

13- Brasil, Ministério da Saúde. Protocolo de manejo clínico do coronavírus (covid- 19) na atenção primária à saúde. Brasília, DF: Ministério da Saúde; 2020e.

14- Januzi FF, Cintra FA. Atividades de lazer em idosos durante a hospitalização. Rev Esc Enferm USP 2006 [citado em 15 mar 2020]; 40(2):179-87. Available http://www.scielo.br/pdf/reeusp/v40n2/04.pdf

15- LabEduca60+ Aprendizagem e Desenvolvimento ao longo da Vida. Envelhecimento saudável em tempos de pandemia. São Paulo; LabEduca60+; 2020.

16- Sommerhalder C, Goldstein LL. O papel da espiritualidade e da religiosidade na vida adulta e na velhice. In: Freitas EV, Py L, Cançado FAX, Doll J, Gorzoni ML. Tratado de geriatria e gerontologia. 3a ed. Rio de Janeiro: Guanabara Koogan; 2011.

17- Brasil, Sociedade Brasileira de Gerontotecnologia (SBGTEC). Gerontecnologia: A tecnologia como ferramenta fundamental para o cuidado à saúde frente à pandemia do covid-19-e futuros. Ribeirão Preto: SBGTEC; $2020 f$. 
18- Secretaria de Justiça e Cidadania (SEJUS/DF). Como cuidar da população idososa. Coronavírus (COVID-19): guia de orientações para prevenção. Brasília, DF: SEJUS; 2020. Available in: http://www.sejus.df.gov.br/coronavirus-sejusdivulga-cartilha-sobre-cuidados-com-idosos/ 19- Brasil, Ministério da Saúde. Gripe (influenza): Causas, sintomas, tratamento, diagnóstico e prevenção. Brasília, DF: Ministério da Saúde; 2020g. Available in: https://www.saude.gov.br/noticias/agenciasaude/46567-comeca-segunda-feira-vacinacaocontra-gripe 20- Brasil, Ministério da Saúde. Portaria SAS/MS no 1298, de 21 de novembro de 2013. Aprova o Protocolo Clínico e Diretrizes Terapêuticas da Doença de Alzheimer. Diário Oficial da União 2013:147-68.

21- Associação Brasileira de Alzheimer (ABRAz). 0 impacto da pandemia do coronavírus nos cuidadores de pacientes com Doença de Alzheimer. Rio de Janeiro: ABRAz; 2020.

22- Baztán, Juan J. Mejorando la hospitalidad del hospital en el paciente anciano con afección aguda. Rev Esp Geriatr Gerontol. 2008;43(3):129-30. DOI: 10.1016/S0211-139X(08)71170-3

23- Eliopoulos C. Enfermagem gerontológica. 5a ed. Porto Alegre: Artmed; 2005.

24- Brasil. Portaria № 758, de 9 de abril de 2020. Define o procedimento para o registro obrigatório de internações hospitalares dos casos suspeitos e confirmados de COVID-19, nos estabelecimentos de saúde públicos e privados que prestam serviços no SUS. Diário Oficial da União 2020;69-C(1):1.

25- Fundação Oswaldo Cruz. Organização emergencial da rede de atenção à saúde no Estado do Rio de Janeiro para enfrentamento da pandemia do novo coronavírus/COVID-19. Rio de Janeiro: Fiocruz; 2020.

26- Kuznier TP, Lenardt MH. The hospitalized elderly patients and the meaning of aging. Rev Enferm Cent O Min. 2011 [citado em 15 mar 2020]; 1(1):70-9. Available in: http://seer.ufsj.edu.br/index.php/recom/article/vie w/29/77

Nota: Produto de atividades realizadas no Projeto de Extensão Cuidando do Idoso e de seu Cuidador (PROECIC - EEAN - UFRJ) e no Programa de Assistência Integral à Pessoa Idosa (PAIPI HESFA - UFRJ).

Recebido em: 21/05/2020

Aprovado em: 13/07/2020

Endereço de Correspondência:

Aline Miranda da Fonseca Marins.

Rua Afonso Cavalcanti, no 275 - Cidade Nova,

CEP: 20071-003 - Rio de Janeiro/RJ -Brasil.

E-mail: alinemiranda@gmail.com 